\title{
Roteiro para a experiência de levitação de um imã repelido por um supercondutor no Ensino de Física
}

\author{
(An outline to the experiment of levitation on a superconductor in Physics teaching)
}

\author{
Fábio Saraiva da Rocha ${ }^{1}$ e Henrique Aita Fraquelli ${ }^{2}$ \\ ${ }^{I}$ Universidade de Santa Cruz do Sul, Departamento de Química e Física, RS, Brasil. \\ Universidade Federal do Rio Grande do Sul, Instituto de Física, Departamento de Física, RS, Brasil. \\ Universidade Estadual do Rio Grande do Sul, RS, Brasil. \\ ${ }^{2}$ Universidade Federal do Rio Grande do Sul, Instituto de Física, Departamento de Astronomia, RS, Brasil. \\ Recebido em 7/4/03; Manuscrito revisado recebido em 25/9/03; Aceito em 28/11/03
}

\begin{abstract}
Propõe-se um roteiro para a demonstração do fenômeno da supercondutividade através da experiência de levitação de um ímã repelido por uma amostra de $\mathrm{YBa}_{2} \mathrm{Cu}_{3} \mathrm{O}_{7}$. A experiência é baseada na manifestação do efeito Meissner, característico de um material no estado supercondutor sob a ação de um campo magnético. A demonstração não requer grande investimento contribuindo com a introdução de tópicos de Física contemporânea na atualização dos currículos. Discute-se a base dos conceitos físicos da supercondutividade envolvidos na levitação do magneto. Ao final é apresentado no apêndice a descrição dos processos envolvidos na fabricação e tratamento térmico de uma amostra do material cerâmico supercondutor de alta temperatura crítica $\mathrm{YBa}_{2} \mathrm{Cu}_{3} \mathrm{O}_{7}$ $\left(\mathrm{T}_{\mathrm{c}} \sim 92 \mathrm{~K}\right)$.
\end{abstract}

Palavras-chave: ensino de Física, experimentação, levitação magnética, supercondutividade.

\begin{abstract}
The aim of this paper is to propose an outline to demonstrate the phenomenon of superconductivity by means of a levitation experiment of a magnet, repelled by a sample of $\mathrm{YBa}_{2} \mathrm{Cu}_{3} \mathrm{O}_{7}$. The experiment is based on the evidence of the Meissner effect, a characteristic property of a superconducting material under the action of magnetic fields. This demonstration of the levitation phenomenon does not imply large expenditures. It helps to introduce contents of the contemporary physics that leads to update curriculum. The main physical concepts involved in the levitation of a magnet and the phenomenon of superconductivity are both discussed here. Finally, the appendix describes the procedures involved in the preparation and thermal treatment of a sample of the ceramic high critical temperature superconducting material $\mathrm{YBa}_{2} \mathrm{Cu}_{3} \mathrm{O}_{7}\left(\mathrm{~T}_{\mathrm{c}} \sim 92 \mathrm{~K}\right)$.
\end{abstract}

Keywords: Physics teaching, experimentation, magnetic levitation, superconductivity.

\section{Introdução}

O fenômeno da supercondutividade foi descoberto [1] em 1911 pelo físico holandês Heike Kammerlingh Onnes, três anos após sua façanha de liquefação do gás hélio que ocorre na temperatura de 4,2 K. Onnes observou que a resistência elétrica de uma amostra de mercúrio desaparecia completamente em temperaturas inferiores a 4 K. Desde sua descoberta, a supercondutividade despertou grande interesse no mundo científico acadêmico e tecnológico. Essa importância pode ser medida pelo número de quatro prêmios Nobel concedidos aos pesquisadores que atuaram brilhantemente na área. Num material supercondutor as cargas elétricas negativas podem fluir livremente sem haver perdas de energia por calor, caracterizando a ausência do efeito Joule. Outra manifestação característica de um supercondutor é o efeito Meissner que consiste na exclusão do fluxo magnético de dentro do material.

O problema com o desenvolvimento tecnológico da supercondutividade tem sido sempre as baixas temperaturas necessárias para mantê-la. Contudo, a partir de 1986,

Enviar correspondência para Fábio Saraiva da Rocha. E-mail: darocha@ if.ufrgs.br. novos materiais cerâmicos supercondutores [2], baseados em óxidos de cobre, foram descobertos. Estes materiais possuem temperaturas de transição para o estado supercondutor consideravelmente mais altas que as dos supercondutores metálicos convencionais como o nióbio, alumínio, o estanho, o chumbo, o índio e algumas ligas intermetálicas. Atualmente, consegue-se o recorde para a temperatura crítica de transição supercondutora $\mathrm{T}_{c} \sim 153 \mathrm{~K}$ num composto do sistema $\mathrm{Hg}-\mathrm{Ba}-\mathrm{Ca}-\mathrm{Cu}-\mathrm{O}$ [3] quando submetido a pressão de $150 \mathrm{kbar}$.

Os recentes desenvolvimentos na área de supercondutividade tem grande importância do ponto de vista das aplicações práticas em tecnologia de ponta, que se tornam mais atraentes na medida que em que alcançamos temperaturas críticas mais elevadas. Em países tecnologicamente mais avançados tem sido comum o desenvolvimento de aplicações baseadas na supercondutividade tais como: construção de sensores magnéticos ultra sensíveis usados para sensoriar campos magnéticos devidos à correntes elétricas inclusive aquelas da atividade cardíaca e cerebral, bobinas geradoras de campos magnéticos intensos necessários para o diagnóstico médico por ressonância magnética, construção de motores elétricos compactos com até 
$97,7 \%$ de eficiência e até $5000 \mathrm{HP}$ de potência, trens que flutuam a poucos centímetros dos trilhos alcançando velocidades acima de $500 \mathrm{~km} / \mathrm{h}$, novos dispositivos eletrônicos mais compactos e com baixíssima dissipação de calor, entre outras.

Visto que a temperatura de ebulição do nitrogênio líquido é de aproximadamente $77 \mathrm{~K}$, em pressão ambiente, e dado o baixo custo deste líquido refrigerante, a observação do fenômeno da supercondutividade pode ser alcançada com relativa facilidade realizando a experiência de levitação magnética. Este experimento se mostra bastante útil para a ilustração do fenômeno de exclusão do campo magnético do interior do supercondutor pelo efeito Meissner.

\section{Aspectos básicos da supercondutividade}

A seguir, discutiremos as propriedades fundamentais da supercondutividade: resistividade nula e efeito Meissner. A idéia aqui é desenvolver os temas com o enfoque qualitativo e conceitual, evitando-se um tratamento matemático muito extenso. Alguns textos [4,5] são indicados para os que desejarem aprofundar-se no assunto.

\subsection{Resistividade nula e temperatura crítica supercondutora $\left(\mathbf{T}_{\mathrm{c}}\right)$}

Os elétrons fracamente ligados aos íons da rede cristalina de um material metálico, quando sujeitos a um campo elétrico originam uma corrente elétrica. Este fluxo ordenado de cargas sofre espalhamento devido a impurezas ou imperfeições no arranjo periódico de íons e às vibrações térmicas que mantêm os íons oscilando em torno de suas posições de equilíbrio. A resistividade elétrica resulta destes efeitos. De um modo geral, um metal na temperatura ambiente tem resistividade elétrica pequena mas não nula. Quando diminuímos a temperatura observamos uma queda na resistividade elétrica pois favorecemos uma diminuição na amplitude das deformações da rede. Mas o que acontece se a temperatura baixar tanto que se aproxime do limite da temperatura absoluta nula? Esta pergunta instigou muito os físicos no início do século passado. Willian Thomson (o Lord Kelvin da escala de temperatura absoluta) previu erroneamente que os próprios elétrons "congelariam" na temperatura nula propiciando assim uma resistividade infinita. Para Onnes, a resistividade elétrica deveria cair lentamente chegando a zero na temperatura nula pois as vibrações dos íons do metal cessariam. Se considerarmos um metal puro e sem imperfeições deveremos ter o comportamento esperado por Onnes. Como não podemos fabricar uma amostra perfeita, teremos sempre uma resistência residual quando nos aproximamos da temperatura absoluta nula. Num supercondutor, contudo, vemos a resistividade indo a zero numa temperatura finita (acima do zero absoluto), chamada temperatura crítica $\mathrm{T}_{\mathrm{c}}$, como mostra a Fig. 1.

A aplicação de um campo magnético a um supercondutor causa uma queda na temperatura $T_{c}$ em que a resistência se anula. Submetendo a um campo suficientemente intenso a supercondutividade desaparece e o material transita para seu estado normal. Este campo é chamado de

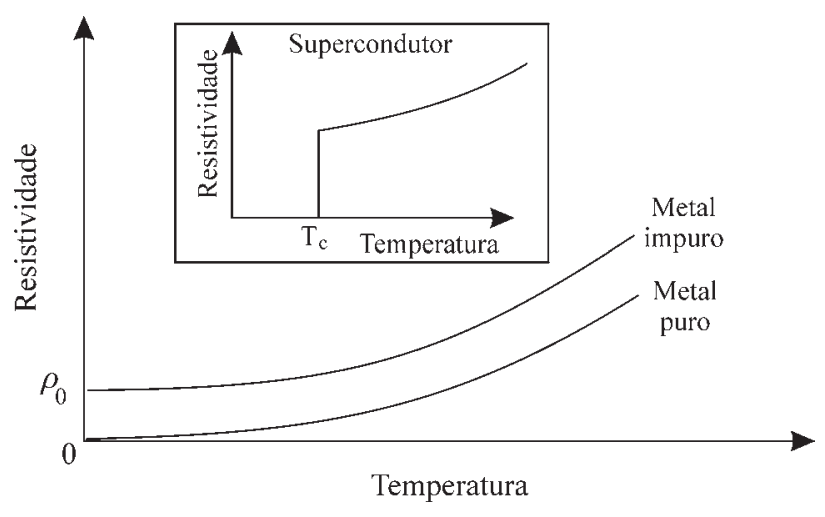

Figura 1 - Comportamento resistivo de um metal puro e de um metal contendo impurezas e ou falhas na estrutura cristalina. O detalhe gráfico inserido mostra o comportamento distinto de um supercondutor, em que a resistividade se anula abaixo da $\mathrm{T}_{\mathrm{c}}$.

crítico $\mathrm{H}_{c}$ e varia como função da temperatura. Observa-se também que, dependendo do valor de densidade de corrente que se quer passar pelo material, a supercondutividade é suprimida devido ao campo magnético por ela mesmo produzido na superfície da amostra. Assim sendo, podemos afirmar que supercondutividade e magnetismo são efeitos mutuamente excludentes.

De acordo com a teoria microscópica BCS - que rendeu o prêmio Nobel de Física em 1972 a Bardeen, Cooper e Schrieffer [6] - os elétrons num supercondutor, em uma temperatura abaixo da $\mathrm{T}_{c}$, estão acoplados aos pares. Este acoplamento é provocado pela interação destes elétrons com a rede de íons do material. De forma sucinta, o que ocorre nesta interação é que um elétron interage com a rede e a perturba. A rede perturbada interage com outro elétron, de maneira que ocorre uma atração efetiva entre os dois. Esta atração supera a repulsão coulombiana e os dois elétrons formam um estado ligado, que possui spin total nulo, denominado par de Cooper. Mais precisamente, um elétron se deslocando pelo material, distorce ligeiramente a rede, que por sua vez, gera uma concentração transiente de carga positiva efetiva. Outro elétron que estiver próximo poderá ser atraído formando assim um par com o primeiro. No estado supercondutor, os pares de Cooper estão correlacionados, atuando em conjunto. Dessa forma, não há dissipação de energia por efeito Joule ao passarmos uma corrente elétrica por um supercondutor pois não existem colisões entre os elétrons e a rede, caracterizando a resistividade nula.

Um fato interessante é que as correntes induzidas num anel supercondutor persistem por muitos anos sem diminuírem de intensidade, mesmo sem haver nenhuma fonte de energia presente no circuito. Esta característica já está sendo usada para armazenamento e fornecimento de energia durante os picos de demanda em cidades de países tecnologicamente mais avançados.

Nas Tabelas 1, 2 e 3 são listados alguns materiais e seus valores da $\mathrm{T}_{\mathrm{c}}$. Estes valores foram obtidos das referências $[1,2,3,4,5,6,7,8,13$ e 14], citadas neste trabalho.

Importante também é relatar que a supercondutividade não é exclusividade apenas de sistemas metálicos e 
Tabela 1 - Alguns metais e seus respectivos valores da $\mathrm{T}_{\mathrm{c}}$. É interessante citar que no $\mathrm{Au}, \mathrm{Ag}$ e $\mathrm{Cu}$, bons condutores elétricos, não foi observado supercondutividade.

\begin{tabular}{ccccc}
\hline & $\mathrm{Rh}$ & $\mathrm{W}$ & $\mathrm{U}$ & $\mathrm{Ti}$ \\
& $0,00033 \mathrm{~K}$ & $0,0154 \mathrm{~K}$ & $0,2 \mathrm{~K}$ & $0,4 \mathrm{~K}$ \\
Metais $\left(T_{c}\right)$ & $\mathrm{Th}$ & $\mathrm{Sn}$ & $\mathrm{Hg}$ & $\mathrm{Ta}$ \\
& $1,3 \mathrm{~K}$ & $3,7 \mathrm{~K}$ & $4,15 \mathrm{~K}$ & $4,5 \mathrm{~K}$ \\
& $\mathrm{~V}$ & $\mathrm{~Pb}$ & $\mathrm{Tc}$ & $\mathrm{Nb}$ \\
& $5,4 \mathrm{~K}$ & $7,2 \mathrm{~K}$ & $7,8 \mathrm{~K}$ & $9,25 \mathrm{~K}$ \\
\hline
\end{tabular}

Tabela 2 - Alguns compostos intermetálicos supercondutores e seus respectivos valores de $T_{c}$. Até o advento das cerâmicas supercondutoras, descobertas em 1986, o recorde da $\mathrm{T}_{\mathrm{c}}$ estava com a liga $\mathrm{Nb}_{3} \mathrm{Ge}$.

\begin{tabular}{lccc}
\hline & $\mathrm{PbSb}$ & $\mathrm{HoMo}_{6} \mathrm{~S}_{8}$ & $\mathrm{Ni}_{2} \mathrm{NbAl}$ \\
& $1,5 \mathrm{~K}$ & $1,8 \mathrm{~K}$ & $2,1 \mathrm{~K}$ \\
Compostos & $\mathrm{Tl}_{2} \mathrm{Hg}_{5}$ & $\mathrm{SnSb}$ & $\mathrm{ErRh}_{4} \mathrm{~B}_{4}$ \\
intermetálicos & $3,8 \mathrm{~K}$ & $3,9 \mathrm{~K}$ & $8,7 \mathrm{~K}$ \\
$\left(T_{c}\right)$ & $\mathrm{Pb}_{2} \mathrm{Au}$ & $\mathrm{PbMo}_{6} \mathrm{~S}_{8}$ & $\mathrm{YNi}_{2} \mathrm{~B}_{2} \mathrm{C}$ \\
& $7 \mathrm{~K}$ & $15 \mathrm{~K}$ & $15,3 \mathrm{~K}$ \\
& $\mathrm{Nb}_{3} \mathrm{Sn}$ & $\mathrm{Nb}_{3} \mathrm{Ge}$ & $\mathrm{MgB}_{2}$ \\
& $18 \mathrm{~K}$ & $23,4 \mathrm{~K}$ & $39,2 \mathrm{~K}$ \\
\hline
\end{tabular}

cerâmicos, mas existe também em alguns sistemas orgânicos.

\subsection{Efeito Meissner}

Para a compreensão desta sessão o leitor necessita de conhecimentos prévios sobre eletromagnetismo. Antes de abordar o efeito Meissner propriamente dito, vamos lembrar da equação constitutiva para os três vetores magnéticos.

Ao submetermos um material a presença de um campo magnético externo $\vec{H}$ (produzido, por exemplo, por correntes macroscópicas num solenóide), este campo penetra no mesmo. A magnetização $\vec{M}$ é a resposta do material a aplicação do campo $\vec{H}$ e se define como o momento magnético por unidade de volume do material. A indução magnética $\vec{B}$ se relaciona com a magnetização e o campo magnético, através da expressão $\vec{B}=\vec{H}+4 \pi \vec{M}$. Esta fórmula é válida em qualquer ponto do espaço, dentro e fora de um material (no último caso $\vec{M}=0$ ).

Em 1933, W. Meissner e R. Ochsenfeld [7] fizeram experiências com amostras de $\mathrm{Sn}$ e $\mathrm{Pb}$ em temperaturas abaixo da $\mathrm{T}_{c}$, aplicando campo magnético. Constataram que os materiais excluíam as linhas de fluxo magnético do próprio interior, de forma que $\vec{B}=0$. O comportamento observado recebeu o nome de efeito Meissner. Vamos explicar melhor isto: se resfriamos o material a temperatura abaixo da $\mathrm{T}_{\mathrm{c}}$ e então submetemos o mesmo à ação de um campo magnético externo $\vec{H}=\frac{c^{2}}{4 \pi} \vec{B}$ (onde $c$ é a velocidade da luz no vácuo), podemos considerar o mecanismo de aparecimento de uma corrente supercondutora (ou supercorrente) $\vec{j}_{s}$ e da resposta diamagnética $\vec{M}$, que resultam da aplicação deste campo magnético. Supondo que tal campo seja suficientemente fraco para não destruir o estado supercondutor, podemos distinguir os seguintes estágios:

a) Durante a aplicação do campo $\vec{H}$ : o equipamento que fornece este campo (eletroímã) leva um certo tempo $\Delta t$ para estabelecer o valor final, constante, para o campo. Há, pois, uma variação $\frac{\partial \vec{B}}{\partial t}=\frac{4 \pi}{c^{2}} \frac{\partial \vec{H}}{\partial t}$ que provoca, enquanto existir, o aparecimento de um campo elétrico induzido $\vec{E}$, tal que $\nabla \times \vec{E}=-\frac{\partial \vec{B}}{\partial t}$.

b) O campo elétrico transitório induzido $\vec{E}$ provoca o surgimento de uma corrente supercondutora $\vec{j}_{s}$ na sua direção. Esta corrente supercondutora é perene (não cessa mesmo depois que $\frac{\partial \vec{B}}{\partial t}$ e consequentemente $\vec{E}$ se anulem).

c) A corrente $\vec{j}_{s}$ é a responsável pelo surgimento do campo magnético reativo $\vec{H}^{\prime}$, expresso pela equação $\nabla \times \vec{H}^{\prime}=\vec{j}_{s}$. É este campo reativo que gera a resposta magnética do material supercondutor.

d) Esquematicamente pode-se descrever estas etapas que ocorrem durante o intervalo de tempo $\Delta t$, em que o campo externo aplicado $\vec{H}$ atinge seu valor final, através da Fig. 2 (a). A resposta perene do supercondutor está esquematizada na Fig. 2 (b).

e) A resposta magnética $\vec{H}^{\prime}$ do material supercondutor é contrária à ação do campo aplicado $\vec{H}$. Define-se a magnetização $\vec{M}$ do material supercondutor, como: $\vec{H}^{\prime}=4 \pi \vec{M}$. No interior do supercondutor existem, pois, os (a)

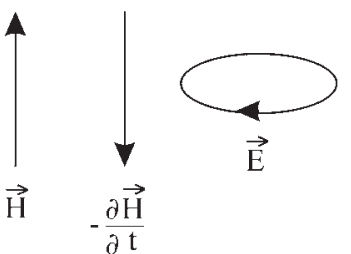

(b)

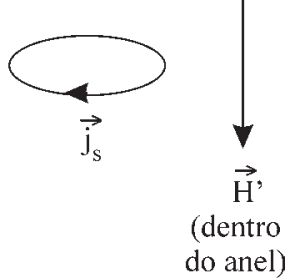

Figura 2 - Esquema mostrando a aplicação de campo magnético sobre uma amostra supercondutora e a resposta do material. Em (a) a situação onde o campo magnético aplicado não atingiu o seu valor final e em (b) somente a resposta perene do supercondutor após o campo magnético externo ter alcançado seu valor constante.

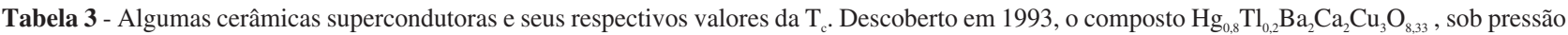
conveniente, é o recordista atual da $\mathrm{T}_{\mathrm{c}}$.

\begin{tabular}{lccc}
\hline & $\mathrm{La}_{1,85} \mathrm{Ba}_{0,15} \mathrm{CuO}_{4}$ & $\mathrm{TmBa}_{2} \mathrm{Cu}_{3} \mathrm{O}_{7}$ & $\mathrm{Bi}_{2} \mathrm{Sr}_{2} \mathrm{Ca}_{2} \mathrm{Cu}_{3} \mathrm{O}_{10}$ \\
Compostos & $40 \mathrm{~K}$ & $101 \mathrm{~K}$ & $110 \mathrm{~K}$ \\
cerâmicos & $\mathrm{Tl}_{2} \mathrm{Ba}_{2} \mathrm{Ca}_{2} \mathrm{Cu}_{3} \mathrm{O}_{10}$ & $\mathrm{Hg}_{0,8} \mathrm{Tl}_{0,2} \mathrm{Ba}_{2} \mathrm{Ca}_{2} \mathrm{Cu}_{3} \mathrm{O}_{8,33}$ & $\mathrm{Hg}_{0,8} \mathrm{Tl}_{0,2} \mathrm{Ba}_{2} \mathrm{Ca}_{2} \mathrm{Cu}_{3} \mathrm{O}_{8,33}$ \\
$\left(T_{c}\right)$ & $127 \mathrm{~K}$ & $138 \mathrm{~K}$ & $($ sob pressão) \\
& & & $153 \mathrm{~K}$ \\
\hline
\end{tabular}


campos $\vec{H}$ (aplicado) e $\vec{H}^{\prime}=4 \pi \vec{M}$ (reativo), tais que o vetor indução magnética $\vec{B}$ se anule $\vec{B}=\vec{H}+4 \pi \vec{M}=0$ ou seja, $-\vec{H}=4 \pi \vec{M}$ (ver Fig. 3).

O efeito Meissner mostra que um supercondutor não é idêntico a um condutor perfeito que obedece às leis do eletromagnetismo de Maxwell. Um condutor perfeito só consegue reagir a uma variação de fluxo magnético no seu interior. Um supercondutor, por sua vez, reage a variação de fluxo magnético ou simplesmente à presença de um campo magnético estático, excluíndo-o de dentro do material se $T \leq T_{C}$. Assim, se aplicamos um campo magnético a um material supercondutor em seu estado normal $\left(\mathrm{T}>\mathrm{T}_{c}\right)$ e o resfriamos sob a ação deste campo, veremos ocorrer uma exclusão abrupta do fluxo magnético $(\vec{B} \rightarrow 0)$ do interior do material quando $\mathrm{T}=\mathrm{T}_{\mathrm{c}}$. Para mantermos o estado supercondutor é importante que o campo magnético não ultrapasse o valor crítico $H_{C}$ que destrói a supercondutividade.

A Fig. 4 apresenta um resumo das características marcantes da supercondutividade.

II.

Os supercondutores são classificados em tipo I e tipo

Num supercondutor tipo I sujeito a campos magnéticos fracos observa-se o estado Meissner (onde o sistema reage a presença do campo externo gerando correntes superficiais que anulam a indução magnética $\vec{B}$ ) até que o campo aplicado sobre a amostra alcance um valor crítico

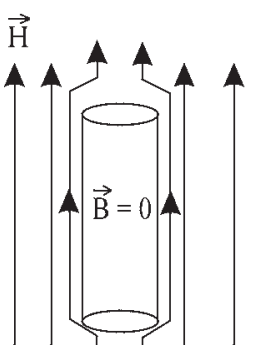

(a)
$\overrightarrow{\mathrm{H}}$

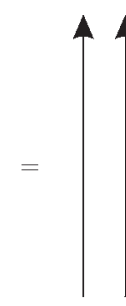

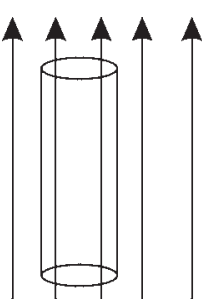

(b)
$4 \pi \overrightarrow{\mathrm{M}}$

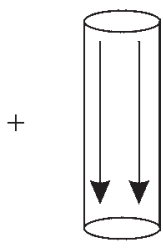

(c)
Figura 3 - Esquema mostrando os campos $\vec{B}, \vec{H}$ e o vetor magnetização $4 \pi \vec{M}$ de uma amostra cilíndrica supercontudora, longa e orientada na direção do campo aplicado.

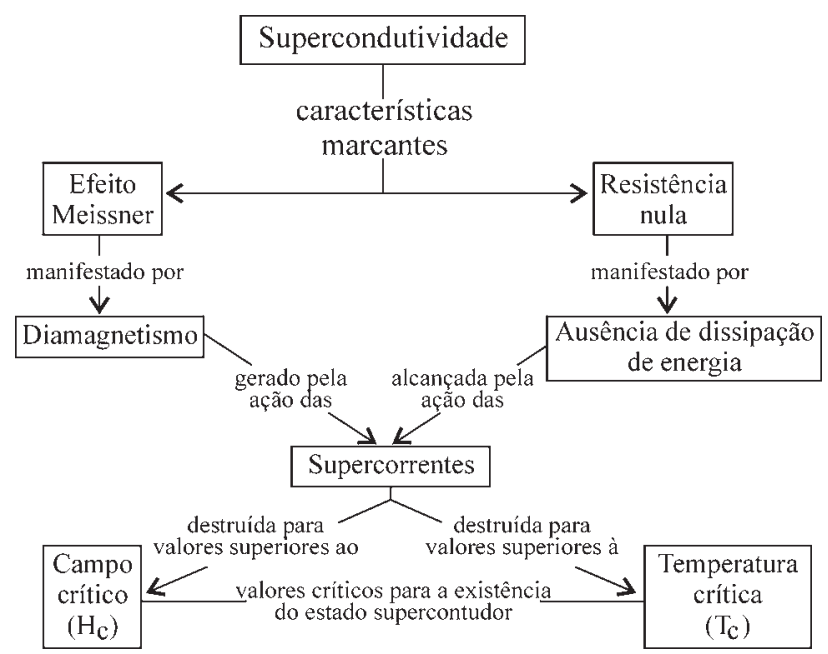

Figura 4 - Diagrama resumindo as principais características da supercondutividade.
$H_{c}$. Os efeitos de campos intensos, com magnitudes próximas ao valor crítico, dependem fortemente da forma da amostra. Na realidade, uma amostra que tenha fator de forma desmagnetizante não desprezível e que esteja próxima de uma transição magnética apresentará um campo magnético inomogêneo em seu interior. Sendo assim, o campo crítico $H_{c}$ será atingido em algumas regiões antes que em outras, gerando a coexistência de domínios supercondutores e normais que caracterizam o estado intermediário. É importante ressaltar que, por exemplo, devido ao fator de forma, numa amostra supercondutora do tipo I, o campo no seu interior será maior ou igual ao campo magnético externo aplicado [8].

O supercondutor de tipo II, apresenta dois campos críticos, $H_{c 1}$ e $H_{c 2}$. Até alcançarmos o campo crítico inferior $H_{c 1}$, o material apresenta-se no estado Meissner, porém, se superamos este valor crítico, que pode ser de dezenas ou centenas de Gauss, o fluxo magnético penetra parcialmente na amostra, permitindo que o sistema mantenha suas propriedades de supercondutividade até que o campo atinja o valor crítico superior $H_{c 2}$. Este último campo pode chegar à casa de centenas de Tesla ( 1 Tesla $=10^{4}$ Gauss) para as cerâmicas supercondutoras como o $\mathrm{YBa}_{2} \mathrm{Cu}_{3} \mathrm{O}_{7}$. Para valores entre $H_{c 1}$ e $H_{c 2}$, o efeito Meissner é incompleto, e se estabelece na amostra o que se chama de estado misto com a criação de uma rede de vórtices de fluxo magnético quantizado que atravessam o material. No interior dos vórtices a supercondutividade é suprimida.

A fim de ajudar o leitor a compreender a magnitude dos campos magnéticos citados acima, colocamos abaixo alguns valores para fins de comparação:

a) campo magnético terrestre: 0,5 Gauss;

b) campo no interior de um solenóide: 100 Gauss;

c) campo na superfície de um ímã de disco rígido: 3000 Gauss;

d) campo no entreferro de um motor elétrico: 15000 Gauss.

A Fig. 5 mostra um diagrama que ajuda a compreender a classificação dos supercondutores.

A amostra de $\mathrm{YBa}_{2} \mathrm{Cu}_{3} \mathrm{O}_{7}$ a ser usada na demonstração da levitação magnética é conseguida através do procedimento descrito no apêndice deste artigo. Na impossibilidade de fabricação da amostra supercondutora, pode-se adquirir o conjunto de materiais para a experiência via importação. Infelizmente o mercado brasileiro ainda não dispõe do conjunto destes materiais que podem ser encontrados com muita facilidade, por exemplo, em mercados da Europa e América do Norte por um valor a partir de 39 dólares [9].

\section{Roteiro para a demonstração da levitação}

Além da amostra de $\mathrm{YBa}_{2} \mathrm{Cu}_{3} \mathrm{O}_{7}$, precisamos dos seguintes materiais:

- Um ímã permanente, na forma de um disco circular, que poderá ser de $\mathrm{NdFeB}, \mathrm{SmCo}$, ou outra composição que gere um campo magnético forte. Um magneto normalmente é encontrado no mecanismo de relógios que utilizam cristal de quartzo com motor de passo. 


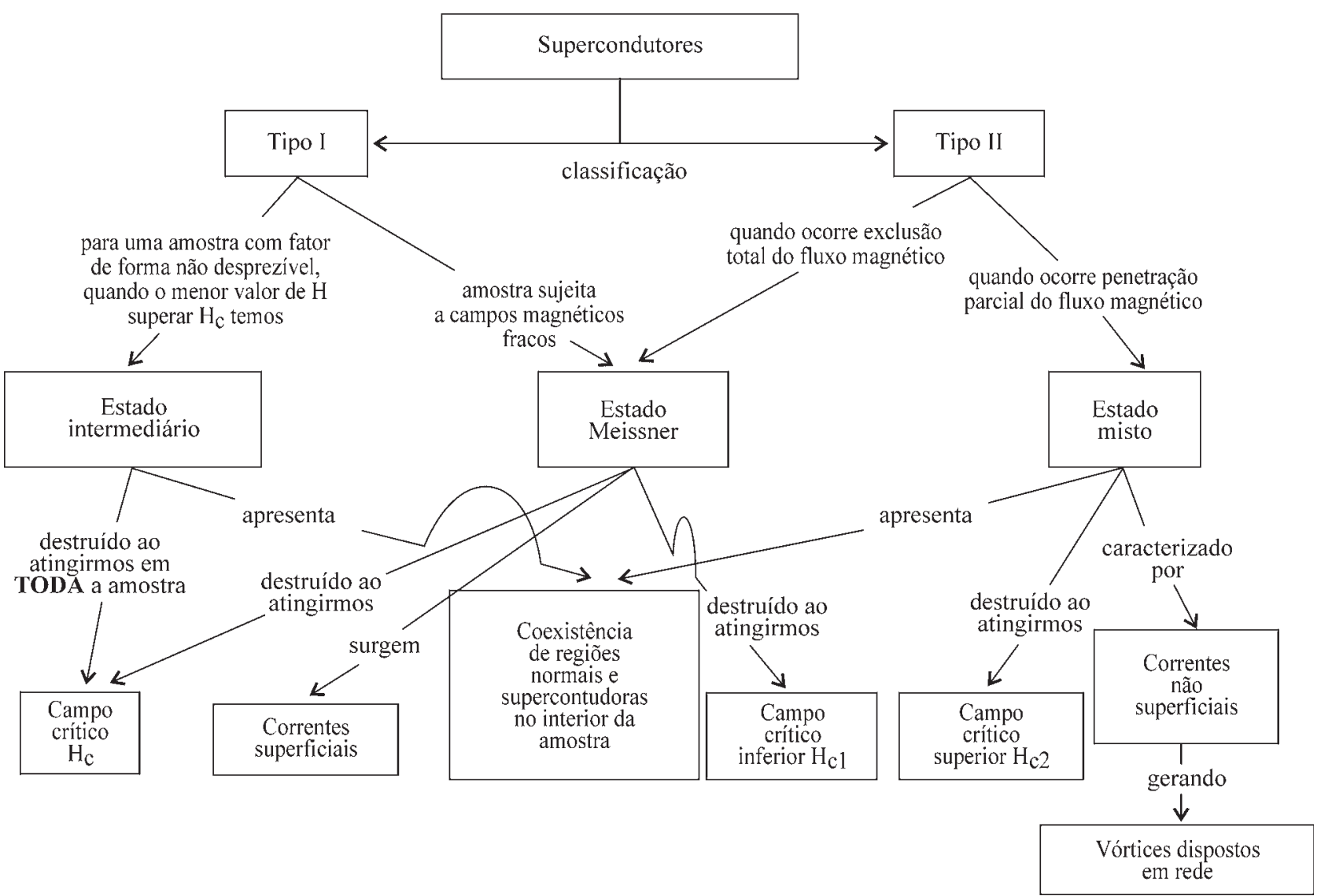

Figura 5 - Diagrama para a classificação dos supercondutores.

- Um reservatório, feito de material isolante térmico (por exemplo: isopor), para conter o líquido refrigerante (nitrogênio líquido). $\mathrm{O}$ isopor, sendo um ótimo isolante térmico, diminui a taxa de evaporação do nitrogênio.

- Um pedestal que serve de suporte para a amostra supercondutora. É desejável que este suporte seja confeccionado com material bom condutor de calor, como por exemplo o latão, para facilitar a termalização da amostra supercondutora.

- Nitrogênio líquido.

- Pinça feita de material não magnético e bússola.

A Fig. 6 mostra um esquema do arranjo experimental usado na demonstração.

\subsection{Passos para a demonstração}

1) Monte o arranjo experimental mostrado na Fig. 6, tomando bastante cuidado com as amostras supercondu-

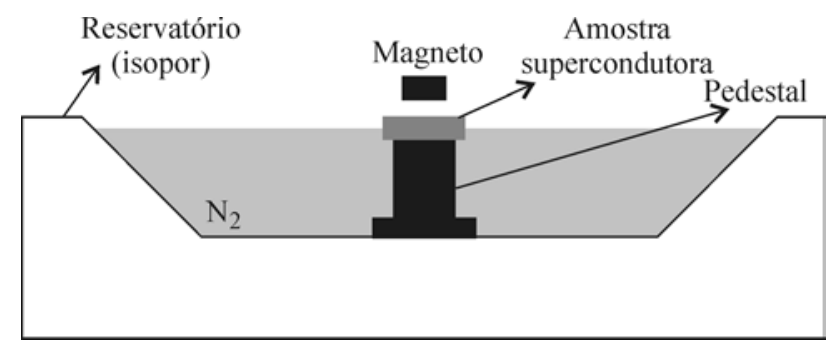

Figura 6 - Esquema do arranjo experimental usado na demonstração da levitação magnética. No equilíbrio, a força peso tem igual direção e intensidade que a força magnética. toras que normalmente são bastante frágeis e não suportam choques.

2) Derrame, com cuidado, o líquido criogênico dentro do reservatório até a altura aproximada da metade da pastilha supercondutora. O líquido borbulha durante alguns minutos, enquanto "rouba" energia interna da pastilha supercondutora, termalizando o conjunto na temperatura de $77 \mathrm{~K}$, abaixo da $\mathrm{T}_{\mathrm{c}^{\circ}}$. Após a termalização, a necessidade de reposição de nitrogênio será menor.

Importante: não deixe o ímã perto do supercondutor durante o resfriamento, pois a amostra supercondutora prende as linhas de campo magnético no seu interior, comportando-se como um ímã que poderá inclusive atrair o magneto.

3) Traga o magneto e coloque-o suavemente sobre o supercondutor. Este deverá levitar. Interessante aqui é levantar o supercondutor com a pinça e verificar a repulsão mútua com o ímã. Para provar que o magneto está levitando, passe um pedaço de papel no vão entre o magneto e o supercondutor. Outro efeito interessante pode ser conseguido com um torque sobre o ímã. Basta a aplicação de uma pequena força sobre a borda do disco para ele entrar em rotação, e girar indefinidamente.

4) Importante completar o nível do líquido refrigerante, periodicamente, para que o supercondutor não transite para o estado normal, o que interromperá a levitação.

5) Estendendo a experiência pode-se mostrar as correntes persistentes que aparecem na superfície de um supercondutor, associadas a resistividade nula. Para isso, 
retira-se o magneto de cima do supercondutor. De acordo com a lei de Faraday, a mudança do fluxo magnético devido a retirada do ímã induz correntes no supercondutor que se opõem a variação de fluxo magnético. Aproxima-se uma bússola da superfície do supercondutor e vê-se que esta alinha-se com o campo gerado pelas correntes persistentes. Retira-se, em seguida, a amostra com a bússola em cima e deixa-se aquecer. Quando o material transitar para o estado normal a bússola voltará a se orientar pelo campo magnético terrestre.

6) É possível também ilustrar, numa etapa posterior, a repulsão entre o supercondutor e o magneto. Para isso, retire o supercondutor do banho líquido e deixe-o aquecer. Recoloque-o dentro do banho junto com o ímã. Assim, o supercondutor é resfriado na presença de campo magnético o que faz com que ele retenha o fluxo magnético no seu interior, devido a impurezas ou imperfeições da amostra supercondutora, passando a se comportar como um ímã com pólos norte e sul. A partir disso é fácil mostrar a atração dos pólos, suspendendo a amostra supercondutora abaixo do magneto.

A Fig. 7 estabelece um diagrama que ilustra os aspectos físicos envolvidos na demonstração da experiência de levitação.

Tenha muito cuidado com a manipulação do nitrogênio pois o contato com a pele causa queimadura profunda. Nunca permita que respingue nos olhos!

\subsection{Explicando o fenômeno da levitação}

Quando aproximamos o magneto do $\mathrm{YBa}_{2} \mathrm{Cu}_{3} \mathrm{O}_{7}$, em estado supercondutor, ocorre o aparecimento de supercorrentes na superfície do material. Estas supercorrentes geram um campo magnético que se opõe ao campo magnético externo, tornando nula a indução magnética dentro do supercondutor $\vec{B}=\vec{H}+4 \pi \vec{M}$. Na ausência de um campo ordenador, dentro do material, os pares de Cooper são mantidos. Os dois campos, o do magneto e o do supercondutor, causam uma repulsão, como dois pólos magnéticos do mesmo sinal. O supercondutor passa a agir como um espelho magnético. A levitação ocorre pois a força magnética, na superfície do supercondutor, é maior que a força

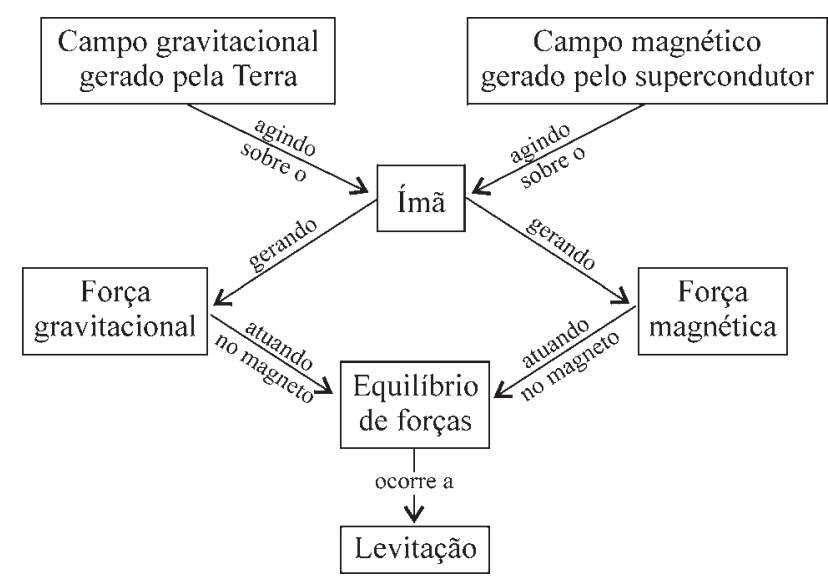

Figura 7 - Diagrama ilustrando os aspectos físicos envolvidos na demonstração da experiência da levitação magnética. peso, levando o ímã a subir até encontrar o ponto de equilíbrio.

A Fig. 8 mostra o comportamento das linhas de indução do magneto quando este está próximo do $\mathrm{YBa}_{2} \mathrm{Cu}_{3} \mathrm{O}_{7}$, na temperatura acima da $\mathrm{T}_{\mathrm{c}}$, portanto, no estado normal.

Na Fig. 9, vê-se a exclusão das linhas de fluxo magnético pela amostra de $\mathrm{YBa}_{2} \mathrm{Cu}_{3} \mathrm{O}_{7}$, agora numa temperatura abaixo da $T_{c}$.

\section{Conclusões}

A partir de um aparato experimental simples, sem a necessidade de um laboratório, é fácil demonstrar que o peso do ímã fica equilibrado pela força magnética. Através da exclusão do campo magnético, que não pode penetrar no supercondutor, produz-se a levitação. A demonstração deste fenômeno pode ser usada como ponto inicial para posteriores aprofundamentos dos conceitos físicos envolvidos na supercondutividade, como a resistividade nula e o efeito Meissner. Esta abordagem de ensino experimental, pro-

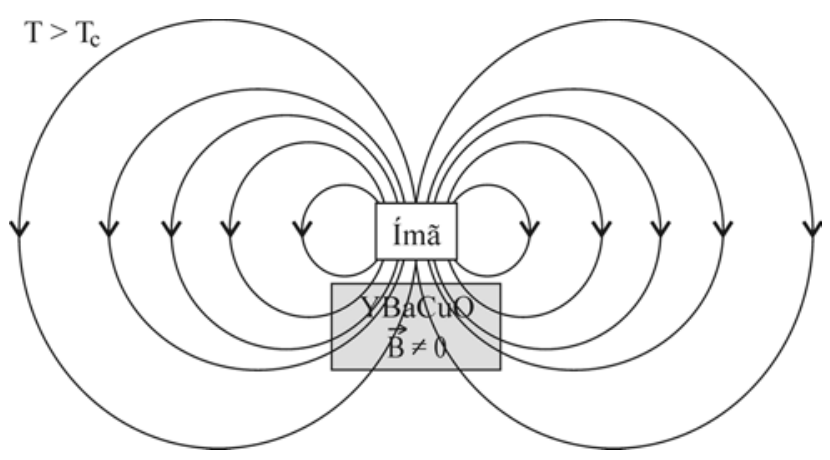

Figura 8 - Esquema das linhas de fluxo magnético geradas pelo magneto. Nota-se que as linhas podem penetrar no interior da amostra de $\mathrm{YBa}_{2} \mathrm{Cu}_{3} \mathrm{O}_{7}$ se $\mathrm{T}>\mathrm{T}_{\mathrm{c}}$. Figura adaptada do artigo original de Ostermann et al. [10].

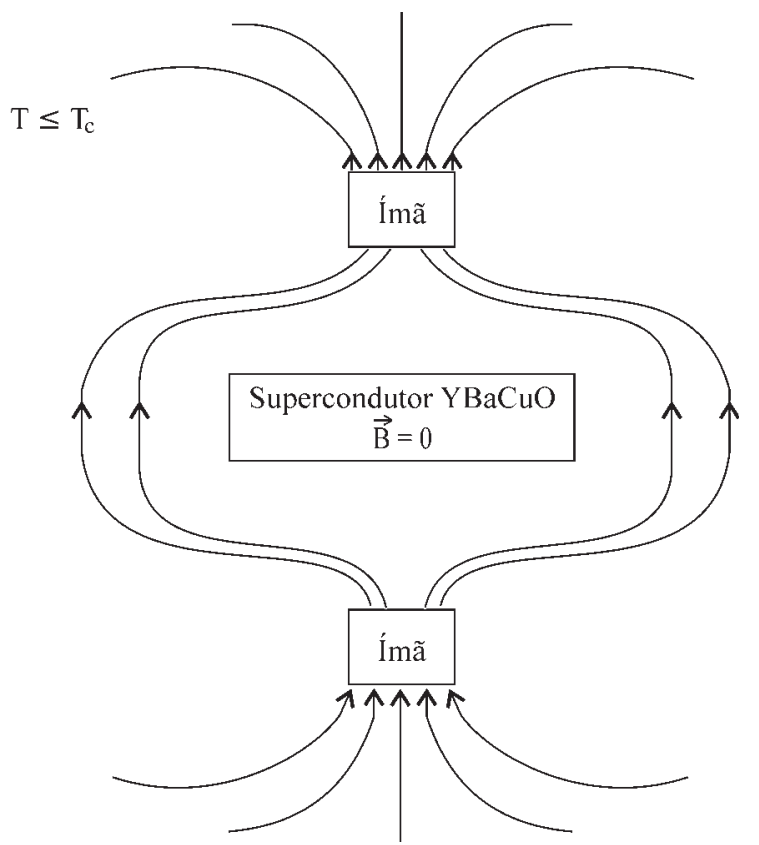

Figura 9 - Esquema das linhas de campo magnético geradas por magnetos, numa situação de efeito Meissner completo. Nota-se que as linhas não penetram no interior da amostra de $\mathrm{YBa}_{2} \mathrm{Cu}_{3} \mathrm{O}_{7}$ se $\mathrm{T} \leq \mathrm{T}_{\mathrm{c}}$. Figura adaptada do artigo original de Ostermann et al. [10]. 
picia ao professor o inicio de uma troca de conhecimentos com sua classe, estimulando os processos de ensino e aprendizagem.

A introdução do tema supercondutividade servirá para melhorar a divulgação de tópicos de Física contemporânea nos currículos atuais [10,11], proporcionando ao aluno contato com tecnologias consideradas de fronteira do conhecimento humano.

\section{Agradecimentos}

Agradecemos aos Professores Delmar E. Brandão e Jacob Schaf do Laboratório de Resistividade do Instituto de Física da UFRGS pela revisão da obra e pelas sugestões apresentadas.

\section{Referências}

[1] H.K. Onnes, Commun. Phys. Lab. Univ. Leiden, 120, 119, 122 (1911).

[2] T.G. Bednorz, K.A. Múller, Z. Phys. B 68, 189 (1986).

[3] C.W. Chu. et al., Nature 356, 323 (1993).

[4] A.C. Rose-Ines, Introduction to Superconductivity. Oxford: Pergamon. (1988).

[5] M. Cyrot, D. Pavuna, Introduction to Superconductivity and High-T Materials (World Scientific, Singapore, 1992).

[6] J.C. Bardeen, L. Cooper, J.R. Schrieffer, Phys. Rev. 106, 162 (1957).

[7] W. Meissner, R. Ochsenfeld, Naturwiss 21, 787 (1933).

[8] F.S. Da Rocha, Estudo Experimental e Teórico das Propriedades Magnéticas e Supercondutoras dos Compostos Borocarbetos da Série $Y\left(\mathrm{Ni}_{1-x} \mathrm{Mn}_{x}\right)_{2} \mathrm{~B}_{2} C \operatorname{com} x=0,0,0,01,0,025$, $0,05,0,10$ e 0,15. Tese de Doutorado, Instituto de Física, UFRGS, 2002.

[9] Colorado Superconductor, Inc. Apresenta os kits de levitação magnética. http://www.users.qwest.net/ csconduc tor/Experiment_Kits/Demonstration\%20Kits.htm. Acesso em 18 de setembro de 2003.

Futurescience, Inc. Apresenta os kits de levitação magnética. http://www.futurescience.com/sc.html. Acesso em 18 de setembro de 2003.

Images SI Inc. Apresenta os kits de levitação magnética. http:// www.imagesco.com/catalog/SuperConductor/SuperConductor.html. Acesso em 18 de setembro de 2003.

[10] F. Ostermann, L.M. Ferreira, C.J.H. Cavalcanti, Rev. Bras. Ens. Fis. 20, 270 (1998).

[11] F. Ostermann, L.M. Ferreira, C.J.H. Cavalcanti, in Textos de Apoio ao Professor de Física n. 8, editado pelo Instituto de Fisica da UFRGS, Supercondutividade: Uma Proposta de Inserção no Ensino Médio (UFRGS, Instituto de Fisica, Porto Alegre, 1998). 74 p. il.

[12] Sigma-Aldrich Co. Apresenta os elementos químicos usados na fabricação da amostra supercondutora http://www. sigmaaldrich.com. Acesso em 18 de setembro de 2003.

Johnson Matthey plc. Apresenta os elementos químicos usados na fabricação da amostra supercondutora http://www. matthey.com. Acesso em 18 de setembro de 2003.

Goodfellow Co. Apresenta os elementos químicos usados na fabricação da amostra supercondutora http://www. goodfellow.com. Acesso em 18 de setembro de 2003.

[13] M. Gurvich, A.T. Fiory, Phys. Rev. Lett. 59, 1337 (1987).

[14] P. Rodrigues Jr., Irreversibilidades Magneticas e Flutuações na Condutividade Elétrica do $\mathrm{YBa}_{2} \mathrm{Cu}_{3} \mathrm{O}_{7-\delta}$. Tese de Doutorado, Instituto de Física, UFRGS, 1994.

\section{Apêndice}

\section{Preparação de uma amostra cerâmica de $\mathrm{YBa}_{2} \mathrm{Cu}_{3} \mathrm{O}_{7}$}

Propomos aqui um conjunto de etapas que podem ser seguidas para o preparo da amostra supercondutora de $\mathrm{YBa}_{2} \mathrm{Cu}_{3} \mathrm{O}_{7}$. Cabe lembrar que além do aparato experimental requerido e dos compostos precursores (que podem ser adquiridos no comércio [12]), o leitor deve possuir uma certa habilidade na preparação de amostras, caso contrário é pouco provável que tenha sucesso nas primeiras tentativas.

Inicialmente fabrica-se uma amostra precursora através de uma reação de estado sólido de três compostos: óxido de ítrio $\left(\mathrm{Y}_{2} \mathrm{O}_{3}\right)$, óxido de cobre $(\mathrm{CuO})$ e carbonato de bário $\left(\mathrm{BaCO}_{3}\right)$, misturados na proporção estequiométrica correta. Por exemplo, para obtermos uma amostra de 4,0 g, os compostos de $\mathrm{Y}_{2} \mathrm{O}_{3}, \mathrm{BaCO}_{3}$ e $\mathrm{CuO}$ são misturados na proporção de (1:4:6), respectivamente. As quantidades de massa ficam 605,2 $\mathrm{mg}$ de $\mathrm{Y}_{2} \mathrm{O}_{3}, 2115,76 \mathrm{mg}$ de $\mathrm{BaCO}_{3} \mathrm{e}$ $1279,04 \mathrm{mg}$ de $\mathrm{CuO}$. Antes de fazer a pesagem dos reagentes, recomenda-se que os três componentes precursores sejam aquecidos e mantidos à temperatura de $150{ }^{\circ} \mathrm{C}$ por $3 \mathrm{~h}$, a fim de eliminar a possibilidade de umidade. Logo após, as quantidades precursoras pesadas são misturadas e trituradas num almofariz até alcançarem a melhor homogeneidade possível, com o pó apresentando cor café. Uma vez homogeneizada, a mistura é acondicionada em um cadinho de alumina $\left(\mathrm{Al}_{2} \mathrm{O}_{3}\right)$, aquecida e mantida a uma temperatura de $950{ }^{\circ} \mathrm{C}$ por $6 \mathrm{~h}$. A seguir, o composto resultante é tirado do forno, novamente macerado e volta para novo recozimento. Nesta segunda etapa, o cadinho contendo o produto da calcinação é colocado dentro de um tubo de quartzo, à temperatura de $950{ }^{\circ} \mathrm{C}$, para receber fluxo constante de oxigênio durante $24 \mathrm{~h}$. Continuando o processo, o composto resultante é novamente macerado e prensado na forma de uma pastilha plana e circular. A pressão aplicada sobre o pó na prensagem é da ordem de $300 \mathrm{kgf} / \mathrm{cm}^{2}$. É conveniente que o diâmetro da pastilha supercondutora seja, no mínimo, o dobro do diâmetro do magneto. Boas dimensões para a pastilha supercondutora são: $20 \mathrm{~mm}$ de diâmetro e $3 \mathrm{~mm}$ de altura. Após a prensagem tem inicio o processo de sinterização da amostra. Nesta etapa a amostra é mantida em fluxo constante de oxigênio, durante $24 \mathrm{~h}$, na temperatura de $950{ }^{\circ} \mathrm{C}$, para otimizar a formação do $\mathrm{YBa}_{2} \mathrm{Cu}_{3} \mathrm{O}_{7-\delta}$. O resfriamento da amostra é feito lentamente $\left(28^{\circ} \mathrm{C} / \mathrm{h}\right)$, em fluxo de $\mathrm{O}_{2}$, até a temperatura de $600{ }^{\circ} \mathrm{C}$ para assegurar que a transição estrutural tetragonal-ortorrômbica, que ocorre em torno de $700{ }^{\circ} \mathrm{C}$, seja completa, garantindo a ausência de precipitados com a estrutura tetragonal não supercondutora [13]. A seguir, a temperatura da amostra é estabilizada em $450{ }^{\circ} \mathrm{C}$ por $24 \mathrm{~h}$ para otimizar a fixação de oxigênio. Chegando ao fim do processo, a estequiometria da amostra é $\mathrm{YBa}_{2} \mathrm{Cu}_{3} \mathrm{O}_{7-\delta}$, onde $\delta$ é pequeno.

Uma análise da qualidade da amostra obtida pode ser feita usando a técnica de difração de raios-X, através do método de pó. A estrutura encontrada deve ser ortorrômbica com valores de parâmetros de rede típicos [14] $\mathrm{a}=3,811 \AA, \mathrm{b}=3,87 \AA$ e c $=11,72 \AA$. 
Para evitar o acúmulo de umidade na pastilha de $\mathrm{YBa}_{2} \mathrm{Cu}_{3} \mathrm{O}_{7}$, a amostra pode ser revestida com uma camada de prata. Este revestimento é feito, por exemplo, com o uso da técnica de Sputtering. No processo, os átomos de prata são arrancados de uma fonte pelo impacto de um feixe de íons e, como vapor, se deslocam até o substrato (amostra) onde se condensam na forma de um filme. A prata tem a propriedade de proteger da umidade e ser permeável ao gás oxigênio. Isto deixa a possibilidade de se aplicar uma nova oxigenação na amostra, se for preciso. Uma outra saída, mais econômica, é revestir a amostra com cola prata que protege da umidade e proporciona um bom contato térmico do material supercondutor com o banho externo.

Ao final do processo a amostra terá uma massa aproximada de $4,5 \mathrm{~g}$. 